\title{
Design and validation of a multiplex PCR protocol for microsatellite typing of Candida parapsilosis sensu stricto isolates
}

\author{
Camino Trobajo-Sanmartín ${ }^{1 \dagger}$, Guillermo Ezpeleta ${ }^{2,3 \dagger}{ }^{+}$Célia Pais $^{4}$, Elena Eraso ${ }^{1}$ and Guillermo Quindós ${ }^{1 *}$ (D)
}

\begin{abstract}
Background: Analysis of polymorphic microsatellite markers (STR) is a helpful genotyping technique to differentiate Candida parapsilosis sensu stricto isolates. The aim of this study is to develop and perform an initial validation of an alternative protocol for the reliable and accurate microsatellite genotyping of C. parapsilosis sensu stricto isolates using high-throughput multiplex PCR. To achieve this, the results obtained using the new protocol were compared to the ones obtained using a previously described reference method. To that end, diagnostic accuracy, informativeness and discrimination parameters were estimated.

Results: Our results showed good concordance between both methods (Kappa index: 0.920), leading to a high sensitivity $(1 ; \mathrm{Cl}(95 \%)(0.991-1))$ and specificity $(1 ; \mathrm{Cl}(95 \%)(0.772-1))$ after the validation of the new protocol. Moreover, the electropherograms profiles obtained with the new PCR scheme showed a high signal to noise ratio (SNR).

Conclusions: The new multiplex protocol is valuable for the differentiation of $C$. parapsilosis sensu stricto, with direct clinical applications. Besides, the new protocol represents a shortening the hands-on time, reducing the sample manipulation (dismissing the possibility of cross-contamination), maintaining the quality of the results (when compared to the ones obtained with the reference method), and helping to the standardization and simplification of the genotyping scheme.
\end{abstract}

Keywords: Candida parapsilosis sensu stricto, Genotyping technique, Microsatellites repeats, Multiplex PCR, Specificity, Reproducibility of results

\section{Background}

In 2005, Tavanti et al. proposed that the fungus Candida parapsilosis could be considered as a genetically related species complex which includes C. parapsilosis sensu stricto, Candida metapsilosis and Candida orthopsilosis [1], being C. parapsilosis sensu stricto the most commonly isolated. However, C. parapsilosis sensu stricto is not a homogeneous species, and therefore, accurate and reliable typing methods are necessary for a better knowledge of this species [1-3]. These typing methods have

\footnotetext{
*Correspondence: guillermo.quindos@ehu.eus

${ }^{+}$Camino Trobajo-Sanmartín and Guillermo Ezpeleta contributed equally to this work.

'Laboratorio de Micología Médica, Departamento de Inmunología, Microbiología y Parasitología, UFI11/25 (Microbios y Salud), Facultad de Medicina y Enfermería, Universidad del País Vasco/Euskal Herriko Unibertsitatea (UPV/EHU), Apartado 699, E-48080 Bilbao, Spain

Full list of author information is available at the end of the article
}

been used for identifying the sources of infection, the chain of transmission and for determining the dissemination of specific strains in the medical environment [4].

Last decades technological innovations allowed the extensive use of microsatellites or Single Sequence Repeats (SSR) in plant and eukaryote genetics studies, using different genotyping approaches ranging from low to high throughput ones, not only in genetic research but also with interesting applications in clinical practice. In fact, microsatellite typing has been described to study genetic relatedness among colonizing and infective strains from diverse geographical locations or even the relatedness of C. parapsilosis isolated from different clinical sources, such as blood or catheters, and from medical or surgical wards $[2,4,5]$. Currently, the microsatellite typing method described by Sabino et al. [5] is one of the reference

(c) The Author(s). 2018 Open Access This article is distributed under the terms of the Creative Commons Attribution 4.0 International License (http://creativecommons.org/licenses/by/4.0/), which permits unrestricted use, distribution, and 
techniques to show genetic relatedness among different clinical isolates of $C$. parapsilosis sensu stricto.

This issue aroused an explosion of alternative protocols to standard procedures giving a large number of genotyping schemes but with no extensive validation by comparing them to the existing ones [6-8]. This work aimed to develop and perform an initial validation of an alternative protocol for the reliable and accurate microsatellite genotyping of Candida parapsilosis sensu stricto isolates using high-throughput multiplex PCR.

\section{Methods}

\section{Microorganisms}

Thirty-three C. parapsilosis sensu stricto blood isolates retrospectively collected during 2010 to 2015 using a convenience sample of 33 patients suffering from invasive candidemia during their hospital stay at La Fe University Hospital (Valencia, Spain). In addition to those clinical isolates, C. parapsilosis sensu stricto ATCC 22019, and ATCC MYA-4646 (CDC317) obtained from the American Type Culture Collection (ATCC, Manassas, VA, USA) were used as positive controls. Moreover, $C$. orthopsilosis ATCC 96139, C. metapsilosis ATCC 96143, Candida albicans ATCC 90028, Candida africana ATCC MYA-2669, Candida dubliniensis NCPF 3949, Candida glabrata ATCC 90030, Candida bracarensis NCYC 3133, Candida nivariensis CBS 9984, Candida tropicalis NCPF 311, Candida krusei ATCC 6258, Candida guilliermondii NCPF 3099 and Lodderomyces elongiosporus ISA1308 (NCPF, National Collection of Pathogenic Fungi, UK; NCYC, National Collection of Yeast Cultures, UK; CBS, Centraalbureau voor Schimmelcultures, Netherland and ISA, Instituto Superior de Agronomía, Portugal) were included as subrogate negative controls to assess the specificity of the multiplex PCR protocol. Besides, PCR grade water (Roche Diagnostics, Germany) was used as negative control for all PCR tests performed.

\section{Culture, isolation and identification}

All isolates were plated onto Sabouraud dextrose agar (Difco, USA) and incubated at $37{ }^{\circ} \mathrm{C}$ for $24 \mathrm{~h}$. Presumptive identification was performed considering colony morphology and color on ChromID Candida (bioMérieux, France) and Candida chromogenic agar (CONDA, Spain) agars and subsequently confirmed using the API $32 \mathrm{C}$ (bioMérieux) auxanogram according to manufacturers. DNA was extracted using the UltraClean ${ }^{\circ}$ Microbial DNA Isolation Kit (MoBio, USA) following the recommendations of the manufacturers. Definitive identification was reached either by amplification of a short portion of the $S A D H$ gene using a conventional RFLP-PCR protocol, as previously described $[1,9]$ or by ITS sequencing when the obtained RFLP-PCR profile from the former technique was inconclusive.
Optimization of multiplex PCR scheme conditions

This step was only performed using the control strains and each prepared multiplex reaction was tested under different primer concentrations (ranging from 0.2 to $0.5 \mu \mathrm{M})$ to ensure the best PCR performance. After this step, another multiplex reaction was tested at different final DNA concentrations (ranging from 10 to $30 \mathrm{ng}$ ) to establish the best amount of yeast genomic DNA needed. Finally, the addition of bovine serum albumin (purified BSA, 100X) (New England Biolabs, USA) to the PCR mastermix to enhance the reaction efficacy was evaluated.

Assay for establishing differences in the polymerase activity Based on previous reports of polymerase inefficiency of prominent slippage phenomena under certain conditions $[10,11]$, we performed a short experiment to evaluate the performance of three different specially designed for multiplex PCR amplification commercial polymerases and their respective mastermixes. To that end, each reference strain was tested in parallel under same amplification conditions using the mentioned mastermixes and its correspondent DNA polymerase. The PCR mastermixes included in the assay were TaKaRa Ex TaqTM Hot Start Version (Takara Bio Inc., Japan), KAPA2G Fast Multiplex PCR kit (Kapa Biosystems, UK) and AmpliTaq Gold $^{\circ}$ DNA Polymerase (Applied Biosystems Inc., USA).

\section{Modified multiplex microsatellite PCR amplification}

All C. parapsilosis sensu stricto were genotyped using CP1, CP4, CP6 and B5 microsatellite markers previously described by Sabino et al. [5]. Because of the rather low annealing temperatures of the designed primers, a multiplex touchdown PCR protocol was chosen to prevent (or minimize) the appearance of unspecific (or non-desirable) PCR products. The amplification reaction had a final volume of $25 \mu \mathrm{l}$ containing $15 \mathrm{ng}$ of yeast genomic DNA, 1X PCR buffer, 1X BSA (100X), 1.25 U of DNA polymerase, $1.5 \mathrm{mM} \mathrm{MgCl} 2,0.4 \mu \mathrm{M}$ of each primer, and $0.2 \mathrm{mM}$ deoxynucleoside triphosphates (dNTPs) and the amplification touchdown PCR protocol was performed in a C 1000TM Thermal Cycler (Bio-Rad, USA).

Briefly, PCR protocol had two differentiated phases. In the first step, annealing temperature of $60{ }^{\circ} \mathrm{C}$ gradually decreased $0.35{ }^{\circ} \mathrm{C}$ per cycle until it reached $55{ }^{\circ} \mathrm{C}$. The second phase was the same as the latter except for three minor modifications, which were a slight increase in the number of cycles (from 14 to 19), a fixed annealing temperature of $55{ }^{\circ} \mathrm{C}$ (see Fig. 1 for more details).

\section{Fragment size determination}

Once the PCR protocol was optimized, 33 C. parapsilosis sensu stricto blood isolates were genotyped using the multiplex scheme proposed in this work and the 


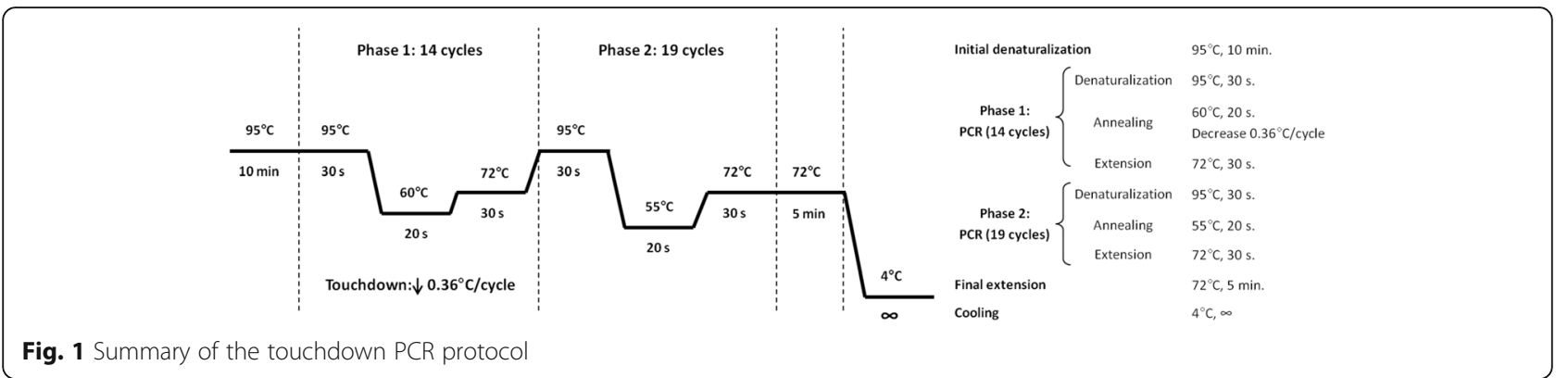

singleplex PCR protocol described by Sabino et al. [5]. For each PCR product size determination, each tested allele forward primer was labeled with a different fluorochrome: 5' 6-Fluorescein (56-FAM) for CP1, 5' MAX (NHS Ester) (5MAXN) for CP4, 5' 5-TAMRA ${ }^{\text {tx }}$ (Azide) (55-TAMK) for CP6, and finally $5^{\prime}$ Rhodamine $\operatorname{Red}^{\mathrm{m}}-\mathrm{X}$ (NHS Ester) (5RhoR-XN) for B5 (IDT, Belgium). One microliter of each obtained amplification product was mixed with $8.6 \mu \mathrm{l}$ of $\mathrm{Hi}-\mathrm{Di}$ formamide and $0.4 \mu \mathrm{l}$ of the internal size standard (GenScan ${ }^{\mathrm{Tm}}$ 500 LIZ $^{\circ}$ Size Standard; Applied Biosystems). This mixture was heated for $5 \mathrm{~min}$ at $95{ }^{\circ} \mathrm{C}$ and immediately cooled to $4{ }^{\circ} \mathrm{C}$ to ensure DNA detachment. After denaturalization, the samples were run on an $A B I$ $\mathrm{PRISM}^{\circ}$ 3130xl Genetic Analyzer (Applied Biosystems) and the final size of the obtained PCR products was determined using the PeakScanner software (version 1.0). The same software was used for the estimation of the number of repeats in each processed allele by direct comparison of the relative size of the clinical isolate to the defined for $C$. parapsilosis sensu stricto reference strains.

\section{Genotype definition and data analysis}

The microsatellite genotypes were defined on the unique combination of alleles obtained for the four loci analyzed and considering that the size differences observed at one or more loci defined different genotypes.

The identification of similarities between genotypes was achieved by the constructions of a minimum spanning tree using R statistical software (v.3.1.0). Besides, to represent the relationship between all the $C$. parapsilosis sensu stricto genotypes obtained, a phylogenetic tree was performed using the POPTREE software. Basically, the phylogenetic tree was inferred from the allele frequency data obtained from the studied samples, was performed using the neighbor-joining method or the unweighted pair-group method with arithmetic mean (UPGMA). Additionally, a bootstrap test was implemented for evaluating the robustness of the results [12].

Besides, we also calculated other parameters of each microsatellite marker considered in this study which are linked to the microsatellite informativeness content and their discrimination power such as the polymorphic information content (PIC), the Simpson index, the heterozygosity and the entropy $[13,14]$.

Finally, an estimation of sensibility, specificity, and the Kappa index was performed to estimate not only the diagnostic characteristics of each microsatellite detection protocol but also the agreement among the results obtained after the microsatellite amplification using each compared PCR protocol. All the statistical procedures were performed using the $\operatorname{Stata}(\mathrm{R})$ and $\mathrm{R}$ statistical software (v. 12 and 3.1.0 respectively). The associations between categorical variables were studied using a chisquared test or Fisher's exact test when necessary.

\section{Ethical issues}

This study does not involve human participants, human data or human tissue. The authors solely used C. parapsilosis sensu stricto strains from different repositories or collections to fulfill the objectives of the study. Although some of the strains used for validation came from a clinical origin, no processing of primary samples was made during the experimental work and therefore, the need for ethics approval and consent to participate was unnecessary according to the Spanish Biomedical Research Law and other European Union regulations. However, a formal approval was asked to the Ethical and Research Committee of the University of the Basque Country to ensure that all the issue research was in accordance with the legal and ethical requests prior to its beginning (Ethics Committee of the Universidad del País Vasco/Euskal Herriko Unibertsitatea UPV/EHU, Bilbao, Spain, reference number CEIAB M30_2015_248).

\section{Results}

\section{Redesign and optimization of original PCR protocol}

Despite the several approaches implemented along with the literature to establish a successful microsatellite based genotyping scheme, we focused on the optimization and restructuring of the original PCR protocol proposed by Sabino and coworkers [5] converting it from a singleplex approach to a multiplex one, avoiding the redesign of the initial primer pairs. 
Table 1 Main characteristics, pros and cons between four C. parapsilosis sensu stricto microsatellite genotyping protocols published in the literature compared and the one described in this work $(N=5)$

\begin{tabular}{|c|c|c|c|c|c|}
\hline Characteristic & Sabino et al. [5] & $\begin{array}{l}\text { Diab-Elschahawi } \\
\text { et al. [6] }\end{array}$ & Reiss et al. [7] & Vaz et al. [8] & $\begin{array}{l}\text { Trobajo-Sanmartín } \\
\text { et al. (This study) }\end{array}$ \\
\hline PCR scheme used & Singleplex ${ }^{\mathrm{b}}$ & Multiplex & Multiplex & Multiplex & Multiplex \\
\hline Redesign of the original primers & NA & Yes & Yes & Yes & No \\
\hline $\begin{array}{l}\text { Number of primers pairs used for each } \\
\text { PCR reaction }\end{array}$ & 1 & 3 & 1 & 4 & 4 \\
\hline $\begin{array}{l}\text { Total number of PCR reactions needed } \\
\text { for a complete STR analysis }\end{array}$ & 3 & 2 & 5 & 1 & 1 \\
\hline $\begin{array}{l}\text { Number of dyes used in each genotyping } \\
\text { reaction }\end{array}$ & 3 & 3 & 1 & 3 & 4 \\
\hline Evaluation of the sensitivity and specificity ${ }^{a}$ & NA & No & No & No & Yes \\
\hline $\begin{array}{l}\text { Evaluation of the microsatellite informativeness } \\
\text { parameters }\end{array}$ & Yes & Unknownc & Unknown ${ }^{c}$ & Unknown ${ }^{\mathrm{b}}$ & Yes \\
\hline $\begin{array}{l}\text { Total time elapsed to obtained results } \\
\text { (in minutes) }\end{array}$ & 138 & 79 & 197 & 138 & 79 \\
\hline $\begin{array}{l}\text { Approximate cost }{ }^{\mathrm{d}} \text { estimated of the primer } \\
\text { pairs used in each protocol (in euros) }\end{array}$ & 5,60 & 15,72 & 7,00 & 9,26 & 12,76 \\
\hline $\begin{array}{l}\text { Approximate total cost }{ }^{\mathrm{d}} \text { estimated for the } \\
\text { complete STR analysis of one sample (in euros) }\end{array}$ & 15,58 & 22,53 & 20,77 & 14,00 & 17,45 \\
\hline
\end{tabular}

NA Not applicable

${ }^{\text {a }}$ Taking the original protocol published by Sabino and coworkers as the gold standard

${ }^{b}$ One of the four reactions could be multiplexed using three different dyes

'Probably the authors did the evaluation of the microsatellite informativeness parameters, but there is no clear reference to that subject in their manuscript

${ }^{d}$ Prices are referred to Spain. We included the DNA extraction, AmpliTaq Gold ${ }^{\circledast}$ PCR kit with magnesium chloride and PCR buffer, PCR plate (96-well) and primer prices to estimate the total cost per sample analyzed using each protocol

Table 1 summarizes the main the characteristics, advantages and disadvantages of different successful $C$. parapsilosis sensu stricto microsatellite genotyping protocols published along the literature compared to the one proposed in our study. The optimization and redesign strategy mentioned earlier implied the evaluation and subsequent election of the two cornerstones of the PCR reaction: the polymerase and primer concentration.

Table 2 summarizes the results obtained during the modified protocol optimization including the sensitivity, specificity and positive predictive value for each polymerase enzyme tested in this study. Our results pointed out that the use of different sort of polymerases could affect to the PCR result. In our experience, the Ampli$\mathrm{Taq}^{\bullet}$ Gold polymerase was the only one of those tested that showed values for both sensitivity and specificity equal to $100 \%$.

Furthermore, we found that there were false positive results (non-specific bands) when we used the KAPA2G and Takara mastermixes. Besides, based on our findings, among all the concentrations tested, we found that the $0.4 \mu \mathrm{M}$ final concentration of each allele primer pair lead to the best PCR results. Using this primer concentration, all PCR products obtained by the multiplex protocol showed the same intensity.

\section{Samples genotyping results}

Table 3 reflects the microsatellite genotyping results for the four loci considered under the two conditions tested in our work. The obtained microsatellite typing results

Table 2 Sensitivity, specificity and predictive values of the multiplex protocol using different polymerases $(N=47)$

\begin{tabular}{|c|c|c|c|c|c|c|c|c|}
\hline Polymerase & FP & FN & $\mathrm{TP}$ & TN & $\begin{array}{l}\text { Sensitivity } \\
\text { (Cl 95\%) }\end{array}$ & $\begin{array}{l}\text { Specificity } \\
\text { (Cl 95\%) }\end{array}$ & $\begin{array}{l}\text { PPV } \\
\text { (Cl 95\%) }\end{array}$ & $\begin{array}{l}\text { NPV } \\
\text { (Cl 95\%) }\end{array}$ \\
\hline \multirow[t]{2}{*}{ TaKaRa® Ex Taq Hot Start Version } & 12 & 0 & 35 & 0 & $100 \%$ & $0 \%$ & $74.47 \%$ & NA \\
\hline & & & & & (90.1\%. 100\%) & $(0.0 \% .26 .5 \%)$ & $(59.7 \% .86 .1 \%)$ & (NA) \\
\hline \multirow[t]{2}{*}{ KAPA 2G Fast Multiplex PCR kit } & 12 & 0 & 35 & 0 & $100 \%$ & $0 \%$ & $74.47 \%$ & NA \\
\hline & & & & & (90.1\%. 100\%) & $(0.0 \% .26 .5 \%)$ & $(59.7 \% .86 .1 \%)$ & $(\mathrm{NA})$ \\
\hline \multirow[t]{2}{*}{ AmpliTaq ${ }^{\oplus}$ Gold DNA Polymerase } & 0 & 0 & 35 & 12 & $100 \%$ & $100 \%$ & $100 \%$ & $100 \%$ \\
\hline & & & & & (90.1\%. 100\%) & $(73.5 \% .100 \%)$ & (90.1\%. 100\%) & $(73.5 \% .100 \%)$ \\
\hline
\end{tabular}

FP False positive results, FN False negative results, TP True positive results, TN True negative results, PPV Positive predictive value, NPV Negative predictive value, CI $9595 \%$ confidence interval, NA Not available 
Table 3 Microsatellite fragment analysis after different PCR schemes $(N=35)$

\begin{tabular}{|c|c|c|c|c|c|c|c|c|}
\hline \multirow[t]{2}{*}{ Strain } & \multicolumn{4}{|c|}{ Microsatellite (bp) Sabino's protocol } & \multicolumn{4}{|c|}{ Microsatellite (bp) This study } \\
\hline & $\mathrm{CP} 1$ & $\mathrm{CP} 4$ & CP6 & B5 & $\mathrm{CP1}$ & $\mathrm{CP} 4$ & CP6 & B5 \\
\hline MYA-4646 & $243 / 243$ & $308 / 327$ & $292 / 295$ & $154 / 154$ & $242 / 242$ & $307 / 327$ & $291 / 294$ & $154 / 154$ \\
\hline ATCC 22019 & $244 / 250$ & $306 / 306$ & $292 / 292$ & $132 / 132$ & $242 / 247$ & $307 / 307$ & $293 / 293$ & $136 / 136$ \\
\hline 429 & $239 / 242$ & $312 / 358$ & $250 / 250$ & 139/139 & $239 / 242$ & $310 / 358$ & $250 / 250$ & 139/139 \\
\hline 431 & $236 / 242$ & $309 / 309$ & $265 / 282$ & $133 / 138$ & $236 / 242$ & $307 / 307$ & $264 / 281$ & $133 / 139$ \\
\hline 476 & $222 / 242$ & $308 / 370$ & $265 / 265$ & $133 / 133$ & $236 / 242$ & $307 / 307$ & $264 / 264$ & 139/139 \\
\hline 477 & $239 / 242$ & $327 / 327$ & $264 / 264$ & $135 / 135$ & $239 / 242$ & $326 / 326$ & $264 / 264$ & $134 / 134$ \\
\hline 480 & $222 / 242$ & $364 / 370$ & $268 / 271$ & $120 / 135$ & $222 / 242$ & $362 / 370$ & $267 / 270$ & $120 / 134$ \\
\hline 482 & $242 / 271$ & $352 / 370$ & $291 / 294$ & 133/137 & $242 / 270$ & $351 / 370$ & $290 / 293$ & $132 / 136$ \\
\hline 486 & $222 / 242$ & $373 / 373$ & $306 / 306$ & $135 / 135$ & $222 / 242$ & $372 / 372$ & $305 / 305$ & $134 / 134$ \\
\hline 489 & 239/239 & $308 / 308$ & $259 / 259$ & $154 / 154$ & 239/239 & $307 / 307$ & $258 / 258$ & $152 / 152$ \\
\hline 491 & $242 / 271$ & $370 / 370$ & 269/291 & $132 / 132$ & $242 / 271$ & $369 / 369$ & $290 / 290$ & $132 / 132$ \\
\hline 499 & $242 / 242$ & $361 / 364$ & $267 / 270$ & $120 / 135$ & $242 / 242$ & $360 / 363$ & $267 / 270$ & $119 / 134$ \\
\hline 504 & $237 / 243$ & $309 / 309$ & $265 / 283$ & 133/139 & $236 / 242$ & $307 / 307$ & $264 / 282$ & $132 / 138$ \\
\hline 509 & $239 / 242$ & $394 / 397$ & $270 / 302$ & $133 / 145$ & $239 / 242$ & $390 / 390$ & $270 / 302$ & $132 / 144$ \\
\hline 512 & $216 / 222$ & $373 / 373$ & $305 / 308$ & $135 / 135$ & $216 / 222$ & $372 / 372$ & $305 / 308$ & $134 / 134$ \\
\hline 514 & $222 / 242$ & $372 / 372$ & $309 / 309$ & $135 / 135$ & $222 / 242$ & $372 / 372$ & $308 / 308$ & $134 / 134$ \\
\hline 517 & $217 / 223$ & $373 / 373$ & $306 / 309$ & 135/135 & $216 / 222$ & $372 / 372$ & $305 / 308$ & $134 / 134$ \\
\hline 521 & $222 / 242$ & $370 / 370$ & $271 / 320$ & $135 / 135$ & $222 / 242$ & $369 / 369$ & $270 / 319$ & $134 / 134$ \\
\hline 522 & $222 / 242$ & $370 / 370$ & $320 / 320$ & $135 / 135$ & $222 / 242$ & $370 / 370$ & $320 / 320$ & $134 / 134$ \\
\hline 534 & $239 / 242$ & $379 / 379$ & $288 / 294$ & $135 / 135$ & $239 / 242$ & $379 / 379$ & $288 / 294$ & $125 / 134$ \\
\hline 536 & $222 / 242$ & $370 / 385$ & $273 / 320$ & $135 / 135$ & $222 / 242$ & $369 / 384$ & $273 / 320$ & $134 / 134$ \\
\hline 542 & 239/242 & $324 / 343$ & $253 / 253$ & $163 / 163$ & $239 / 242$ & $323 / 343$ & $253 / 253$ & $163 / 163$ \\
\hline 543 & 239/239 & $355 / 355$ & $273 / 273$ & $111 / 111$ & $239 / 239$ & $354 / 354$ & $273 / 273$ & $110 / 110$ \\
\hline 547 & $239 / 242$ & $379 / 379$ & $250 / 317$ & $135 / 135$ & $239 / 242$ & $379 / 379$ & $250 / 250$ & $134 / 134$ \\
\hline 565 & $239 / 242$ & $352 / 352$ & $273 / 308$ & $111 / 111$ & $239 / 242$ & $351 / 351$ & $273 / 308$ & $110 / 110$ \\
\hline 568 & $222 / 242$ & $373 / 373$ & $305 / 308$ & 135/135 & $222 / 242$ & $372 / 372$ & $305 / 308$ & $134 / 134$ \\
\hline 569 & $222 / 242$ & $370 / 370$ & $318 / 321$ & 135/135 & $222 / 242$ & $369 / 369$ & $317 / 320$ & $134 / 134$ \\
\hline 588 & $222 / 242$ & $370 / 370$ & $320 / 320$ & 135/135 & $222 / 242$ & $369 / 369$ & $320 / 320$ & $135 / 135$ \\
\hline 591 & $222 / 242$ & $370 / 370$ & $276 / 320$ & 135/135 & $222 / 242$ & $369 / 369$ & $276 / 319$ & $135 / 135$ \\
\hline 592 & 239/242 & $400 / 420$ & 270/302 & 133/145 & $239 / 242$ & $400 / 420$ & $270 / 302$ & $133 / 145$ \\
\hline 593 & $219 / 259$ & $308 / 308$ & $267 / 288$ & 153/155 & $219 / 259$ & $307 / 307$ & $267 / 287$ & $153 / 156$ \\
\hline 595 & $222 / 242$ & $370 / 370$ & $270 / 320$ & $135 / 135$ & $222 / 242$ & $369 / 369$ & $270 / 320$ & $135 / 135$ \\
\hline 596 & $242 / 242$ & $361 / 364$ & $267 / 270$ & $120 / 135$ & $242 / 242$ & $360 / 363$ & $267 / 270$ & $120 / 135$ \\
\hline 599 & $236 / 242$ & $308 / 308$ & $264 / 264$ & 139/139 & $236 / 242$ & $307 / 307$ & $264 / 264$ & $139 / 139$ \\
\hline 600 & $242 / 242$ & $361 / 364$ & $267 / 270$ & $120 / 135$ & $242 / 242$ & $360 / 363$ & $267 / 270$ & $120 / 135$ \\
\hline
\end{tabular}

using the protocol described by Sabino et al. [5] compared to the ones using the multiplex PCR protocol proposed in our work, both were identical. This issue lead a high sensitivity (1; CI(95\%) (0.991-1)) and specificity $(1 ; \mathrm{CI}(95 \%)$ $(0.772-1))$ values during the validation step of the new protocol. Furthermore, both techniques showed no amplification in any of the negative control strains (even close-related species such as L. elongiosporus) included in this study. Besides, the ATCC 22019 positive control strain gave the same profile described in the literature after microsatellite fragment analysis (Table 3$)[2,15]$.

Despite these excellent results, the electropherograms obtained frequently showed low-intensity non-specific bands, which were considered as stutter bands due to the polymerase slippage during the PCR (Fig. 2). However, the small amplitude of these artifacts did not 

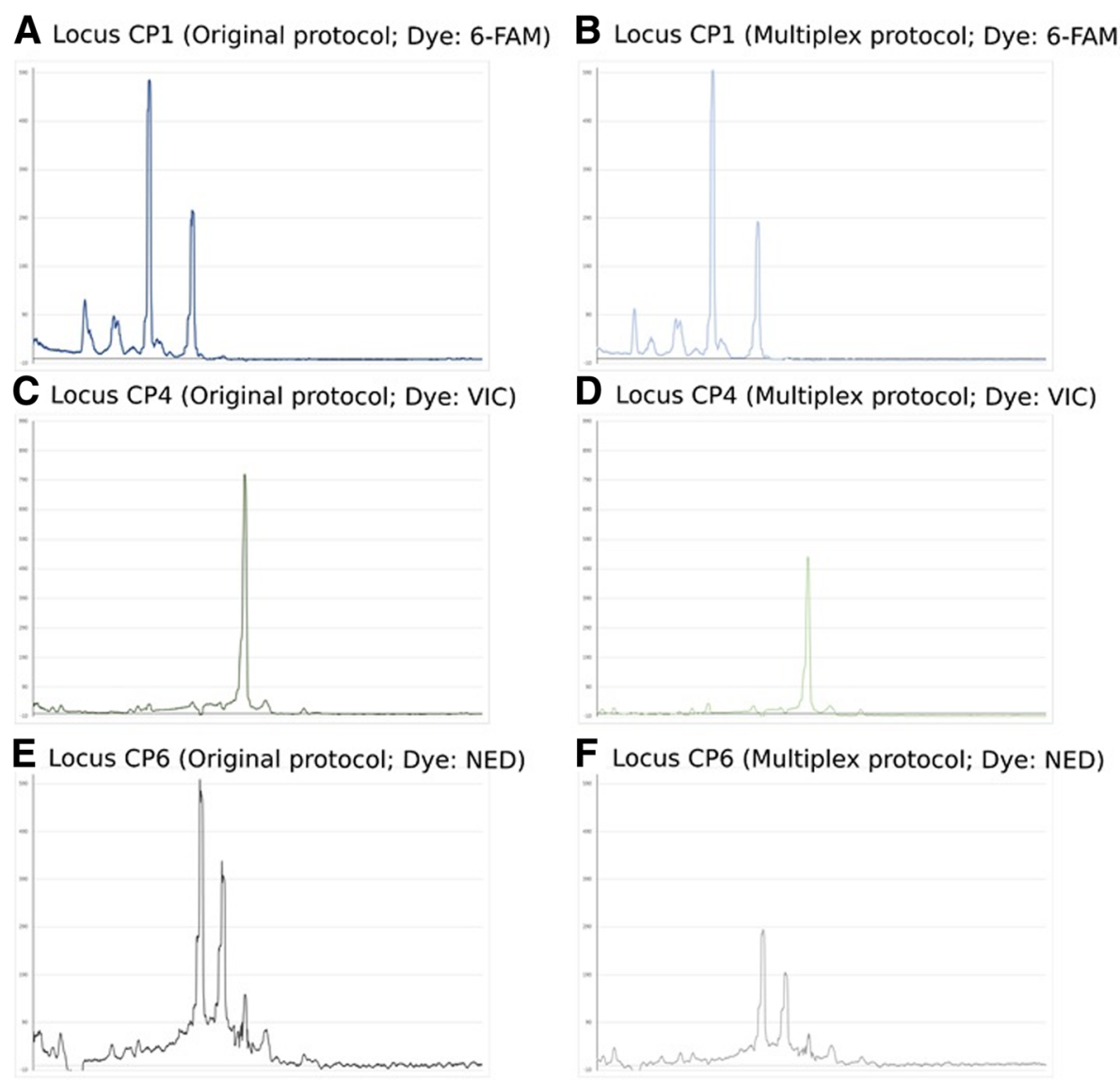

G Locus B5 (Original protocol; Dye: PET)

H Locus B5 (Multiplex protocol; Dye: PET)
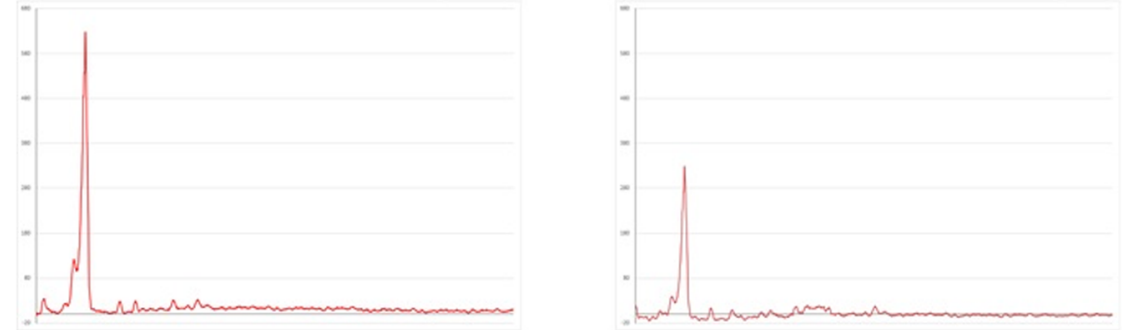

Fig. 2 Example of the electropherograms obtained after genotyping the one of the clinical isolates Candida parapsilosis sensu stricto (clinical isolate 593) using the original simplex protocol described by Sabino and coworkers ( $\mathbf{a}, \mathbf{c}, \mathbf{e}$, and $\mathbf{g})$ and the multiplex one used in this study $(\mathbf{b}, \mathbf{d}, \mathbf{f}$, and $\mathbf{h})$

interfere with the correct identification of the fragment size and subsequently had no impact on the new protocol specificity.

Besides, the similar signal-to-noise ratio of the resulting electropherograms was recorded when compared the new proposed touchdown PCR scheme to the original protocol described by Sabino et al. in 2010 or other slight modifications to that one $[2,4,8,16]$.

To obtain a graphical view of the results mentioned above, we performed a dendrogram based on the microsatellite genotypes identified from the clinical isolates $C$. parapsilosis sensu stricto analyzed using both protocols. This dendrogram is represented in Fig. 3.

\section{Estimation of the information contained in the regions examined}

The obtained estimates of the informativeness parameters investigated are summarized in Table 4. Regarding the observed allele heterozygosis of the analyzed $C$. parapsilosis sensu stricto strains, our results revealed several differences among the analyzed loci. The heterozygosis percentages ranged from $84.85 \%$ for locus CP1 to $27.27 \%$ for locus CP4. However, the heterozygosis rates observed for locus CP6 and locus B5 were $60.61 \%$ and $33.33 \%$, respectively. The discrimination power of each considered allele was concordant with those previously published by Sabino et al. [5]. The 


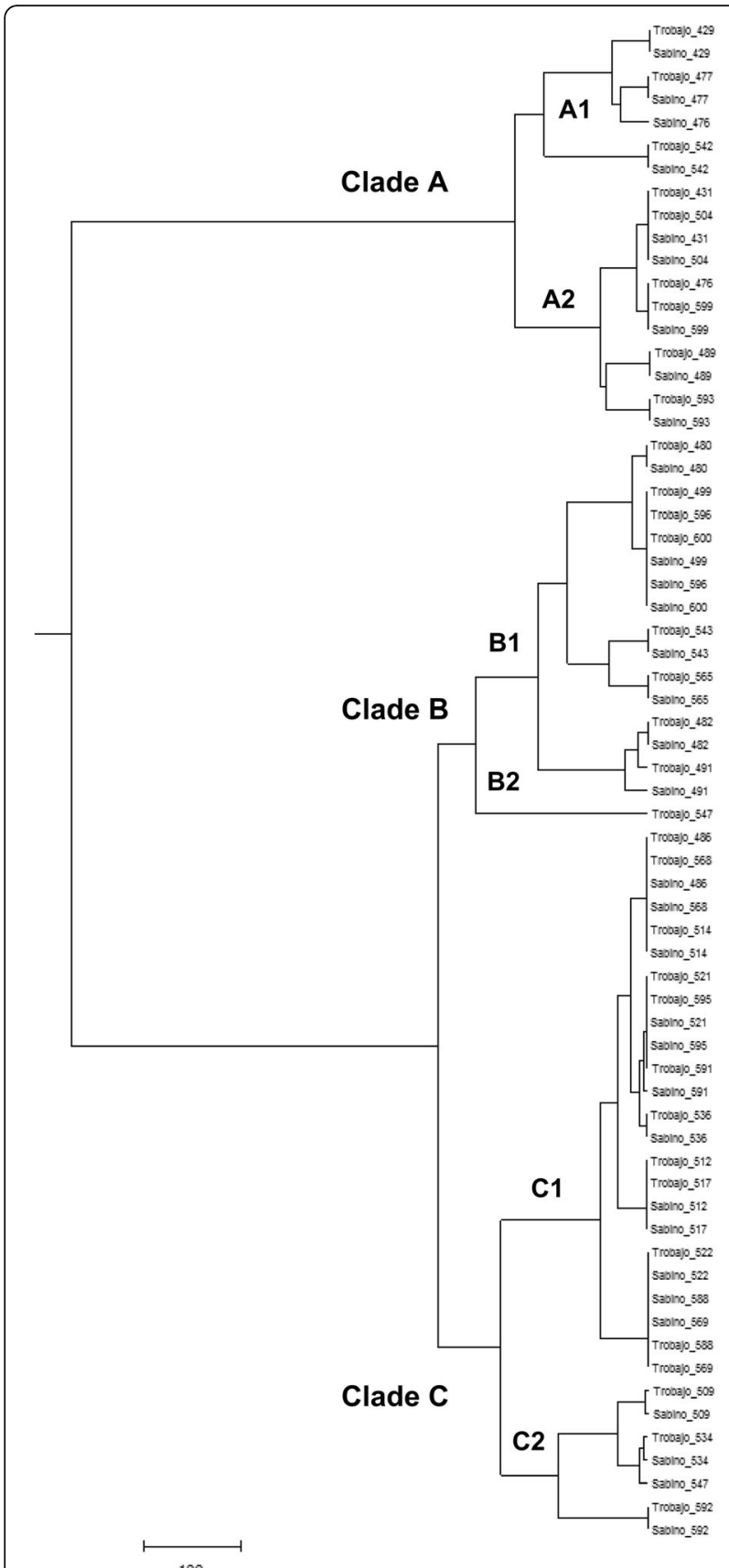

100

Fig. 3 An unweighted pair group method dendrogram based on the microsatellite genotypes identified from the clinical isolates Candida parapsilosis sensu stricto $(N=33)$ analyzed in this study

Simpson index oscillated from 0.702 for CP1 to 0.925 for CP6 marker, which means that the CP1 marker achieved the lowest discrimination power.

\section{Concordance analysis}

Table 5 summarizes the concordance between the improved multiplex protocol and the reference genotyping technique using the direct concordance and the Kappa indices, being both greater than $80 \%$. According to the literature, these results suggest that there is a high concordance level among both genotyping schemes [17].

\section{Discussion}

Several methods, such as isoenzyme analysis, random amplified polymorphic DNA (RAPD), restriction fragment length polymorphism (RFLP) and multilocus sequence typing (MLST), have been described for Candida isolates typing $[1,18,19]$. However, the discriminatory power of some of these typing methods for differentiating $C$. parapsilosis isolates is rather small and many isolates are indistinguishable [3]. In recent days, matrix-assisted laser desorption/ionization timeof-flight mass spectrometry (MALDI TOF-MS) and the analysis of polymorphic microsatellite regions have been described as useful and high discriminatory power techniques for further differentiation of $C$. parapsilosis sensu stricto isolates [3, 20]. However, it seems that MALDI-TOF MS-based typing does not fully correlate with other DNA-based genotyping methods leading to different dendrogram profiles when using protein-based or DNA-based techniques and moderate concordance values between those techniques [21]. Therefore, though MALDI TOF-MS is a reliable technique for identifying isolates at species-level, perhaps more studies are needed to assess its role in fungal genotyping [21,22].

Until recently, microsatellite genotyping is a rather time-consuming technique, because every microsatellite marker must be processed alone. Up to our knowledge, no multiplex PCR protocol following the original scheme proposed by Sabino et al. [5] has been described to that end along with the literature. Recently, Diab-Elschahawi et al. [6] published a PCR protocol using a multiplex approach for $\mathrm{CP} 1, \mathrm{CP} 4$ and CP6 markers redesigning the primers proposed in the original work by Sabino et al. [5, 6]. The disparities between the annealing temperatures of the original primers designed by Sabino and coworkers [5] difficult the amplification of all the loci at the same time, and therefore, other approaches such as primer redesign are necessary. Despite the success of these redesigned primer protocols, we focused a different solution based on optimization and redesign of the PCR protocol (from a singleplex approach to a multiplex one) avoiding the redesign of the initial primer pairs. This solution gave comparable results to the original approach published by Sabino et al. [5] during the validation step with high sensitivity and specificity.

Based on our results, there are several crucial points to consider before getting satisfactory results, being the appropriate polymerase election the most important one when a multiplex PCR protocol is used. Although all the PCR mastermixes tested in our work were explicitly 
Table 4 Discrimination and information parameters for each microsatellite markers. The Simpson index and the observed heterozygosis obtained in the original procedure by Sabino et al. [5] are also included

\begin{tabular}{|c|c|c|c|c|c|c|}
\hline Parameters & CP1 & CP4 & CP6 & B5 & Multiplex & Study \\
\hline Polymorphic information content (PIC) & 0.692 & 0.870 & 0.942 & 0.802 & 0.957 & This study (2016) \\
\hline Simpson Index & 0.724 & 0.879 & 0.944 & 0.816 & 0.959 & \\
\hline Observed heterozygosis & 0.723 & 0.273 & 0.606 & 0.333 & 0.958 & \\
\hline Unbiased estimation of heterozygosis & 0.724 & 0.880 & 0.945 & 0.816 & 0.960 & \\
\hline Entropy (in bits) & 2.450 & 3.647 & 4.430 & 3.179 & 4.688 & \\
\hline Simpson Index & 0.850 & 0.890 & 0.960 & 0.860 & 0.990 & Sabino et al. (2010) [5] \\
\hline Observed heterozygosis & 0.733 & 0.214 & 0.523 & 0.300 & - & \\
\hline
\end{tabular}

fabricated to operate under their best conditions using multiplex PCR protocol, KAPA2G and Takara mastermixes, showed lack of specificity, conditioning their future use in multiplex PCR based $C$. parapsilosis sensu stricto genotyping protocols. The most reliable explanation of the observed results is that the three mastermixes tested had different polymerases in their composition, being the AmpliTaq Gold $^{\oplus}$ the most suitable one to carry out C. parapsilosis sensu stricto microsatellite genotyping using this multiplex PCR scheme.

A total of 35 samples were genotyped to validate the utility of our method in contrast to the one described by Sabino and coworkers. Though our results were concordant with those published previously, we could see slight differences in the estimation of the Simpson index and the observed heterozygosis among Sabino's original data and ours, probably explained because of the differences in the total sample number of strains analyzed in each work.

Finally, the high-quality profiles of the electropherograms obtained using the new multiplex protocol are due to the adoption of a touchdown PCR strategy which improves the profile analysis and prevents misclassification. In a recent review, such schemes are described as a suitable option to increase the specificity of the obtained PCR products without losing sensitivity [23].

There are some limitations in our study such as the small number of strains analyzed in this study and the fact that all of them were isolated from the same clinical source (blood). This issue has probably an impact on the precision of the confidence intervals and the generalization of our informativeness parameters estimates. However, the consistency of our results with those published in the literature suggesting that the possibility of bias is rare.

Despite these limitations, our validation results support that the new protocol seems to be as accurate and reliable as the original one. However, it represents a significant decrease in the turnaround time necessary to get accurate genotyping results compared to other approaches published along with the literature. The main disadvantage the new protocol is that it is slightly more expensive than the original technique in case we use primers labeled with different fluorophores. This limitation could be overcome by using the same fluorophore for those primers targeting loci that have very different sizes (such as CP6 and B5), decreasing the total cost of the technique and increasing its

Table 5 Direct concordance and Kappa indices of the multiplex PCR touchdown protocol

\begin{tabular}{|c|c|c|c|c|c|c|c|}
\hline \multirow[t]{2}{*}{ Multiplex PCR approach genotyping technique (this study) } & \multicolumn{7}{|c|}{ Original genotyping technique [5] } \\
\hline & Clade A1 & Clade A2 & Clade B1 & Clade B2 & Clade C1 & Clade C2 & Total \\
\hline Clade A1 & 3 & 0 & 0 & 0 & 0 & 0 & 3 \\
\hline Clade A2 & 1 & 5 & 0 & 0 & 0 & 0 & 6 \\
\hline Clade B1 & 0 & 0 & 8 & 0 & 0 & 0 & 8 \\
\hline Clade B2 & 0 & 0 & 0 & 0 & 0 & 1 & 1 \\
\hline Clade C1 & 0 & 0 & 0 & 0 & 12 & 0 & 12 \\
\hline Clade C2 & 0 & 0 & 0 & 0 & 0 & 3 & 3 \\
\hline Total & 4 & 5 & 8 & 0 & 12 & 4 & 33 \\
\hline Agreement & Expected agreement & \multicolumn{2}{|c|}{ Kappa index } & \multicolumn{2}{|c|}{ Std. Error of Kappa index } & Z & $p$-value \\
\hline 0.939 & 0.240 & 0.920 & & 0.0917 & & 10.04 & $<0.001$ \\
\hline
\end{tabular}


cost-effectiveness if the researchers decide to adopt the latter option.

\section{Conclusions}

In conclusion, this new protocol is a valuable tool for the differentiation of $C$. parapsilosis sensu stricto isolates, with direct applications to clinical practice and infection control procedures (for example, nosocomial outbreaks). Besides, our protocol helps the standardization and simplification of the existing microsatellite typing systems, improving the quality of data, the sample hands-on time and lab turnaround time to get accurate genotyping results for further clinical or infection control epidemiological studies.

\begin{abstract}
Abbreviations
55-TAMK: TAMRA-tetramethyl-6-rhodamine; 56-FAM: 56-carboxyfluorescein; 5MAXN: Max 557 NHS ester; 5RhoR-XN: Rhodamine Red-X; ATCC: American type culture collection; bp: Base pair; BSA: Bovine serum albumin; CBS: Centraalbureau voor Schimmelcultures; Cl: Confidence interval; DNA: Deoxyribonucleic acid; dNTPs: Deoxynucleotide triphosphates; FN: False negative; FP: False positive; ISA: Instituto Superior de Agronomía; MALDI TOF-MS: Matrix-assisted laser desorption/ionization time-of-flight mass spectrometry; MLST: Multilocus sequence typing; NCPF: National Collection of Pathogenic Fungi; NCYC: National Collection of Yeast Cultures; NPV: Negative predictive value; PCR: Polymerase chain reaction; PIC: Polymorphic information content; PPV: Positive predictive values; RAPD: Random amplified polymorphic DNA; RFLP: Restriction fragment length polymorphism; SADH: Secondary alcohol dehydrogenase; SNR: Signal to noise ratio; SSR: Single sequence repeats; STR: Short tandem repeat; TN: True negative; TP: True positive; U: Units; UPGMA: Unweighted pair-group method with arithmetic mean
\end{abstract}

SI units

${ }^{\circ} \mathrm{C}$ : Celsius degrees; $\mathrm{h}$ : Hour; min: Minute; mM: Millimolar; ng: Nanograms; s: Second; $\mu$ l: Microliters; $\mu$ M: Micromolar

\section{Acknowledgments}

The authors thank Dr. Javier Pemán and Dra. Emilia Cantón (HUP La Fe, Valencia, Spain) for supplying the Candida parapsilosis strains. SGlker technical and human support (UPV/EHU, MICINN, GV/EJ, ESF) is gratefully acknowledged.

\section{Funding}

This work was supported by the Consejería de Educación, Universidades e Investigación of the Gobierno Vasco-Eusko Jaurlaritza [GIC 15/78 IT-990-16] and the Universidad del País Vasco/Euskal Herriko Unibertsitatea [UFI 11/25]. CTS has been supported by a research grant of the Fundación Jesús de Gangoiti Barrera.

\section{Availability of data and materials}

The datasets generated or analyzed during the current study are not publicly available due we have no secure institutional repository and the main results are adequately summarized along with the manuscript. However, additional details are available from the corresponding author on reasonable request and a signed data transfer form. Neither the fragment analysis results nor the electropherograms obtained have been deposited in any genetic database because up to our knowledge there is no opportunity to upload such data in those repositories.

\section{Authors' contributions}

CTS and GE designed the multiplex PCR, conducted the experiments and analyzed the data. GE, CP, EE and GQ participated in the coordination and concept of the manuscript. All authors read and approved the final manuscript.

\section{Ethics approval and consent to participate}

This study does not involve human participants, human data or human tissue. The authors solely used C. parapsilosis sensu stricto strains from different repositories or collections to fulfill the objectives of the study. No processing of primary samples was made during the experimental work and the need for ethics approval and consent to participate was unnecessary according to the Spanish Biomedical Research Law and other European Union regulations. However, a formal approval was asked to the Ethical and Research Committee of the University of the Basque Country to ensure that all the issue research was in accordance with the legal and ethical requests prior to its beginning (Ethics Committee of the Universidad del País Vasco/Euskal Herriko Unibertsitatea UPV/EHU, Bilbao, Spain, reference number CEIAB M30_2015_248).

\section{Consent for publication}

Not applicable.

\section{Competing interests}

We have no specific conflicts of interest related to the current manuscript but declare the following: EE has received grant support from Astellas Pharma and Pfizer SLU. GQ has received grant support from Astellas Pharma, Gilead Sciences, Merck Sharp and Dohme, Pfizer SLU, and Scynexis. He has also been an advisor/consultant to Merck Sharp and Dohme and Scynexis, and has been paid for talks on behalf of Abbvie, Astellas Pharma, Gilead Sciences, Merck Sharp and Dohme, Pfizer SLU, and Scynexis. The authors have not other relevant affiliations or financial involvement with any organization or entity with a financial interest in or financial conflict with the subject matter or material discussed in the manuscript apart from those disclosed above.

\section{Publisher's Note}

Springer Nature remains neutral with regard to jurisdictional claims in published maps and institutional affiliations.

\section{Author details}

'Laboratorio de Micología Médica, Departamento de Inmunología, Microbiología y Parasitología, UFI11/25 (Microbios y Salud), Facultad de Medicina y Enfermería, Universidad del País Vasco/Euskal Herriko Unibertsitatea (UPV/EHU), Apartado 699, E-48080 Bilbao, Spain. ${ }^{2}$ Departamento de Medicina Preventiva y Salud Pública, Facultad de Medicina y Enfermería, Universidad del País Vasco/Euskal Herriko Unibertsitatea (UPV/EHU), Bilbao, Spain. ${ }^{3}$ Servicio de Medicina Preventiva e Higiene Hospitalaria, Complejo Hospitalario de Navarra, Pamplona, Spain. ${ }^{4}$ Centro de Biologia Molecular e Ambiental (CBMA), Departamento de Biologia, Universidade do Minho, Braga, Portugal.

Received: 23 February 2018 Accepted: 11 September 2018 Published online: 29 September 2018

References

1. Tavanti A, Davidson AD, Gow NA, Maiden MC, Odds FC. Candida orthopsilosis and Candida metapsilosis spp. nov. to replace Candida parapsilosis groups II and III. J Clin Microbiol. 2005;43:284-92.

2. Romeo O, Delfino D, Cascio A, Lo Passo C, Amorini M, Romeo D, et al. Microsatellite-based genotyping of Candida parapsilosis sensu stricto isolates reveals dominance and persistence of a particular epidemiological clone among neonatal intensive care unit patients. Infect Genet Evol. 2013;13: $105-8$.

3. van Asbeck EC, Clemons KV, Stevens DA. Candida parapsilosis: a review of its epidemiology, pathogenesis, clinical aspects, typing and antimicrobial susceptibility. Crit Rev Microbiol. 2009;35:283-309.

4. Sabino R, Sampaio P, Rosado L, Videira Z, Grenouillet F, Pais C. Analysis of clinical and environmental Candida parapsilosis isolates by microsatellite genotyping-a tool for hospital infection surveillance. Clin Microbiol Infect. 2015:21(10):954.e1-8

5. Sabino R, Sampaio P, Rosado L, Stevens DA, Clemons KV, Pais C. New polymorphic microsatellite markers able to distinguish among Candida parapsilosis sensu stricto isolates. J Clin Microbiol. 2010;48:1677-82.

6. Diab-Elschahawi M, Forstner C, Hagen F, Meis JF, Lassnig AM, Presterl E, Klaassen $\mathrm{CH}$. Microsatellite genotyping clarified conspicuous accumulation of Candida parapsilosis at a cardiothoracic surgery intensive care unit. J Clin Microbiol. 2012;50:3422-6. 
7. Reiss E, Lasker BA, Lott TJ, Bendel CM, Kaufman DA, Hazen KC, Lockhart SR. Genotyping of Candida parapsilosis from three neonatal intensive care units (NICUs) using a panel of five multilocus microsatellite markers: broad genetic diversity ans a cluster of related strains in one NICU. Infect Genet Evol. 2012;12:1654-60

8. Vaz C, Sampaio P, Clemons KV, Huang YC, Stevens DA, Pais C. Microsatellite multilocus genotyping clarifies the relationship of Candida parapsilosis strains involved in a neonatal intensive care unit outbreak. Diagn Microbiol Infect Dis. 2011;71:159-62.

9. Miranda-Zapico I, Eraso E, Hernández-Almaraz JL, López-Soria LM, CarrilloMuñoz AJ, Hernández-Molina JM, et al. Prevalence and antifungal susceptibility patterns of new cryptic species inside the species complexes Candida parapsilosis and Candida glabrata among blood isolates from a Spanish tertiary hospital. J Antimicrob Chemother. 2011;66:2315-22.

10. Hosseinzadeh-Colagar A, Haghighatnia MJ, Amiri Z, Mohadjerani M, Tafrihi M. Microsatellite (SSR) amplification by PCR usually led to polymorphic bands: evidence which shows replication slippage occurs in extend or nascent DNA strands. Mol Biol Res Commun. 2016;5(3):167-74.

11. Rocha AJ, de Souza Miranda R, Maranguape da Silva Cunha R. Assessment of DNA polymerases in microsatellite amplification assays through PowerPlex ${ }^{\oplus} 16$ BIO system. Biochem Biotechnol Rep. 2014;3:1-8.

12. Takezaki N, Nei M, Tamura K. POPTREEW: web version of POPTREE for constructing population trees from allele frequency data and computing some other quantities. Mol Biol Evol. 2014;31:1622-4.

13. Hunter PR. Reproducibility and indices of discriminatory power of microbial typing methods. J Clin Microbiol. 1990;28:1903-5.

14. Reyes-Valdés MH. Informativeness of microsatellite markers. In: Kantartzi SK, editor. Microsatellites: methods and protocols. Totowa: Human Press; 2013. p. 259-70.

15. Delfino D, Scordino F, Pernice I, Lo Passo C, Galbo R, David A, et al. Potential association of specific Candida parapsilosis genotypes, bloodstream infections and colonization of health workers' hands. Clin Microbiol Infect. 2014:20:0946-51.

16. Barbedo $L S$, Vaz C, Pais C, Figueiredo-Carvalho MH, de Muniz M M, Zancope-Oliveira RM, et al. Different scenarios for Candida parapsilosis fungaemia reveal high numbers of mixed C. parapsilosis and Candida orthopsilosis infections. J Med Microbiol. 2014:64:7-17.

17. Landis JR, Koch GG. The measurement of observer agreement for categorical data. Biometrics. 1977;33:159-74.

18. Tavanti A, Hensgens LAM, Ghelardi E, Campa M, Senesi S. Genotyping of Candida orthopsilosis clinical isolates by amplification fragment lenght polymorphism reveals genetic diversity among independent isolates and strain maintenance within patients. J Clin Microbiol. 2007;45:185-91.

19. van Asbeck E, Clemons K, Markham A, Stevens D. Molecular epidemiology of the global and temporal diversity of Candida parapsilosis. Scand J Infect Dis. 2008;40:827-34

20. Pulcrano G, Roscetto E, lula VD, Panellis D, Rossano F, Catania MR. MALDITOF mass spectrometry and microsatellite markers to evaluate Candida parapsilosis transmission in neonatal intensive care units. Eur J Clin Microbiol Infect Dis. 2012;31:2919-28.

21. Prakash A, Sharma C, Singh A, Kumar Singh P, Kumar A, Hagen F, Govender $\mathrm{NP}$, Colombo AL, Meis JF, Chowdhary A. Evidence of genotypic diversity among Candida auris isolates by multilocus sequence typing, matrixassisted laser desorption ionization time-of-flight mass spectrometry and amplified fragment length polymorphism. Clin Microbiol Infect. 2016;22(3): 277.e1-9.

22. Zautner AE, Masanta WO, Weig M, Groß U, Bader O. Mass spectrometrybased PhyloProteomics (MSPP): a novel microbial typing method. Sci Rep. 2015;5:13431.

23. Korbie DJ, Mattick JS. Touchdown PCR for increased specificity and sensitivity in PCR amplification. Nat Protoc. 2008:3:13-5.

Ready to submit your research? Choose BMC and benefit from:

- fast, convenient online submission

- thorough peer review by experienced researchers in your field

- rapid publication on acceptance

- support for research data, including large and complex data types

- gold Open Access which fosters wider collaboration and increased citations

- maximum visibility for your research: over $100 \mathrm{M}$ website views per year

At BMC, research is always in progress.

Learn more biomedcentral.com/submissions 